\title{
Quantum Dots in Waste Water Treatment- Review Article
}

\author{
Dr. A. Sandy Subala ${ }^{*}$, Dr. K.V.Anand ${ }^{2}$, Mr.S.Antony Sibi ${ }^{3}$ \\ ${ }^{1}$ Senior Lecturer, Faculty in Chemistry, DMI St. Eugene University, Lusaka, Zambia \\ ${ }^{2}$ Lecturer, Faculty in Biochemistry, DMI St. Eugene University, Lusaka, Zambia. \\ ${ }^{3}$ Assistant Lecturer, Faculty in Commerce, DMI St. Eugene University, Chipata campus, Zambia
}

\begin{abstract}
The effluents present in the water resources leads unqualified for various domestic and irrigation purposes. The waste water treatment purely depends upon the volume of contaminants. The objective of the study was to gives a detailed discussion about the chemical methods and different types of QDs involved, examining their effluents removing efficiency, the selectivity and sensitive detection of various chemicals present in the contaminated water bodies.
\end{abstract}

Keywords:- Graphene QDs, CdS QDs, Photolysis, Photoelectron catalysis, photon shielding effect.

\section{INTRODUCTION}

Water is globally considered as most crucial component for the endurance of life, nevertheless the fresh water resources are endangered by human activities. The gradual industrialization discharged innumerable quantity of chemicals leads to various diseases, damages the ecosystem and threatens the global life[1].The released pollutants mixed with ground and surface water impairment the human health and the ecosystem. Moreover, clean and pure water is the fundamental need for the human's healthy life. Thus, it is required to process the polluted water for several purposes like drinking, irrigation and industrial processes. Researchers follow several treatment methods to obtain the fresh water [2]. The presence of pollutant even in low quantity pollutes the water and soil aggravatingly [3]. The hazardous and carcinogenic effect decides the type of pollutants [4]. Organic compounds are the important pollutants released from the manufacturing unit[5]. These organic pollutants deplete the dissolved oxygen cause a risk to human health [6].

It is reality that the heavy metals are nonbiodegradable, likely to cause carcinogenic effects on human beings lead to alter the gene expression and oxidative stress in living organisms by combining with dependent protein [7]. However, the extended use of organophosphorus compounds in agriculture fields percolated into water resources that reduce the grade of water rigorously. The surface water mixed with trace of chemical substances that contaminates the drinking water potential [8]. The spillage of petrochemicals and oil from industry and urban discharge plays a vital role in the contamination of marine water [9]. Most industries need a good amount of fresh water for proper functioning, however the effluents discharged from the industries causes side effects to human health [10].
Owing to this, the quality of the water is declined and it is desirable to inhibit the contaminants by exploitation of affordable and high efficiency water treatment technologies.

Moreover, the most permeative challenge should be preserve theexisting fresh water bodies to meet the growing water demands [11] and in the next forty years the demands on water will increase gradually due to global population [12]. It can be achieved by better water treatment technologies like adsorption, RO purification, UV purification, coagulation and floatation. The working principle of these technologies differs from one another in terms of degree of pollutants, maintenance and operation. During the last two decades significant quantity of research is focussed on the potential application of quantum Dots in water treatment emerged and ignited tremendous research interest due to its unique properties.

\section{QUANTUM DOTS}

The semiconductor nano crystals are recently emerging nano materials best known as Quantum Dots (QD) which have large surface to volume ratio, zero dimensional specific inorganic metals with nano scale size particles having optical and electronic properties in which the excitation of electrons between valence band to conduction band is confined in three spatial directions, which has an ability to produce extreme bright fluorescence that helps to detect the single particle [13, 14, 15]. However, this quantum confinement effect, the outstanding property that increase of band gap energy with decreasing of nanoparticle diameter below certain value makes the quantum dot as artificial atoms and the measure of the energy gap depends on the size of QDs [16].It can be said that, very few number of atoms in QDs separatesthe energy levels in considerable degree, results the existence of atomic like wave functions [17].

Fluorescent carbon based nano material possessing unique electronic properties along with large surface area, reducing and oxidizing ability recognized graphene as a most important component in novel sensing platforms [18, 19]. The electronic properties of graphene has given an origination of graphene based sensors that can detect charge changes in different concentration media there by, helping in identification of surface functional groups and adsorbates at small level [20]. Undoubtedly, in these QDs one can identify abundance surface groups which are capable of strong photoluminescence (PL).The most 
effective light effect of PL is always for a discussion. Since the observed PL centre have Quantum confinement effect conjugated domains and surface edge state encompassed within the structure. The unified effect of the above two states are taken for a greater impecunious of the study of graphene QDs [21].

The UV region of the visible light is the focus of absorbing graphene QDs. The concept of photoluminescence (PL) is stable within the UV light yet, it shows weak photo-bleaching [22].This effect may be due to surface state and quantum size effect of the PL emission in graphene QDs.It has to be understood that the influence of single or multi layers in graphene QD is taken into concern [23], as a PL can also be quenched through selective doping of specific cations, anions or chemical groups [24,25]. Collaborating these features, it is evident that graphene QDs serve as effective sensors for PL detection by "turnoff' model. Further, graphene QDs with intrinsic structures exhibit different selective quenching phenomena there by, making it possible to detect various ions and chemical groups. This concept denote the fact that within the blue green region of the spectrum a striking energy band of PL characteristic of graphene QD is observed [26].

\section{WASTE WATER TREATMENT}

The ionic form of hydrogen sulphide reduces the oxygen concentration in water leads to nervous system damage in human beings. It was required to find out the suitable techniques to treat the water for human and irrigation purposes. Comparatively methods adopted with QDs techniques are excellent water purification performance [27,28]. The selectivity and sensitive detection of $\mathrm{H}_{2} \mathrm{~S}$ in waste water is made possible through the strong bond created with doping element and consecutively followed by reduction which affects the intensity of fluorescence, which is not common ion found in water resource [29].Ag-doped CdS QDs capped by MercaptoAceticAcid (CdSAg-MAA QDs) with size 3.3 $\mathrm{nm}, \mathrm{pH} 5.0$, limit of detection $3 \mathrm{nM}$ and Relative Standard Deviation is $0.54 \%$ served as an effective fluorescence probe due to their exact band edge of $2.4 \mathrm{eV}$ that provides the high photo catalytic activity for enhanced chemical transformation [30]. However, the poor photochemical stability is overcome by doping the $\mathrm{CdS}$ along with the semiconductor of the element. At this stance, thedoping ofCdS QDs with $\mathrm{Ag}$ is taken as a popular and effective example owing to the high exchange current density with low activation energy of $\mathrm{Ag}[31,32]$. At the same time, the co-stabilizing agents increase the detection selectivity for $\mathrm{H}_{2} \mathrm{~S}$ in the waste water. As a major predicament of reality, graphene QDs combined with $\mathrm{Cu}^{2+}$ have been used as the fluorescence probe for sulfide detection [33]. This fluorescence probe is highly selective and sensitive for $\mathrm{S}^{2-}$ in any water sample that contains anionic interferenceswith a limit of detection of $0.10 \mu \mathrm{M}$.
The coloured materials in water was treated through direct UV photolysis and ozonation techniques that study the use of QDs in waste water that take the colouration of the reaction of reactive blue 137 and toluene if it gets to mix in that water.The catalytic potential of the QDs were studied with the assistance of $\mathrm{CdSe} / \mathrm{ZnS}$ core-shells. It was investigated that QDs acts as an additional pollutant rather the catalyst [34].Further the semiconductor QDs $\mathrm{Zn}$ or $\mathrm{Cd}$ exhibits inhibitory effect upon UV radiation.In fact the photon shielding effect of the semiconductor QD is observed as it diminishes the efficiency of photolysis. Moreover, the illumination of semiconductor particle by UV light causes the electrons transferred from the valence band to conduction band that creates an electron-hole pair at the semiconductor nano particle surface. Simultaneously, thePhoto generated electrons are able to react with adsorbed electron acceptor which can be taken as dissolved oxygen from its protonated form.

At an estimated analysis, it is found that 16 $\%$ semiconductor QD consisting of $\mathrm{Cd}$ or $\mathrm{Zn}$ helps to reduce the concentration of the colour after 60 minutes of a reaction. It is observed that the physical properties and reactivity of nano particles vary to a larger extent as a function as a function of size [35, 36]. Ahmad et al investigated the biofouling, major disadvantage of Forward Osmosis for the first time using integrated GQDs with size $3.4-8.8 \mathrm{~nm}$ in polyamide (PA) layer of thin film composite (TFC) membrane in which the water contact angle falling drastically leads to the decrease in solute and water permeability ratio. The resulting Thin Film Nanocomposite (TFC) shows excellent antibacterial behaviour.It is observed that the reference of GQDs integrated with polyamide matrix adds the essence to the hydrophilic attributes of the TFC membrane there by bringing a change to the antifouling attributes that determines the characteristic properties of forward osmosis being done with the bacteria [37]. The anti biofouling property of Escherichia coli showed that the GOQDsPVDF (polyvinylidene fluoride) exhibits excellent biofouling resistance rather than other modified PVDF membranes. The TFC membranes are effectivein biofilm formation when comparedto the graphene oxide membrane [38].

Furthermore, the homogeneous CdTe capsulized wurtzitestructure of ZnOnanorods with band gap 1.5 eVthat is fabricated through layer-by-layer exhibits an excellent photovoltaic effect, serves as a potential matrix for degrade phenol form waste water. The incorporation of CdTe with $\mathrm{ZnO}$ enhances the photocatalytic efficiency of $\mathrm{ZnO}$. This is done through the process ofphotoelectron catalysis which works on strong absorption of visible light creating the degradation efficiency is $75 \%$. The photocurrent efficiency depends on the length of nano rod exclusively. The CdTe$\mathrm{ZnO}$ composite degrade phenols more effective through photoeletrocatalytic technique [39].Besides that, the resultantphotoluminescence (PL)creates quenching that separates the charge carriers between ZnOand CdTe [40, 41] .This leads to optimization of CdS QDs by means of Fractional Factorial Design (FFD) technique which 
enhanced using sodium thiosulfate which serves as a substitute for $\mathrm{TiO}_{2}$ due to the effective photocatalytic activity.The enhanced constituent attribute is the reusing constancy of the coloured compounds under the visible radiation in this process [42].Further,the superoxide and OH serve as the participating intermediates leads to the degradation of dyestuff $[43,44]$.

The reference to fluorescent chemosensors was of great importance as they work upon the ions in solution to a greater degree [45]. The presence of $\mathrm{Fe}^{3+}$ and $\mathrm{Hg}^{2+}$ ions can be easily detected using fluorescent $\mathrm{N}-$ GQDs and $\mathrm{SN}$ GQDsin waste water through solvothermal method. The fluorescent is proportional to thevariation of excitation wavelength but remains stable at a $\mathrm{pH}$ range of $3.5-9.5$, below this limit the fluorescence intensity decreases and above this range it increases [46].

The intriguing properties of carbon QDs degrade the coloured pollutants upon irradiation with infrared and visiblelight [47].Here in as previously referred the low photoluminescence of $\mathrm{CQDs} / \mathrm{TiO}_{2}$ composite nano fibres could remove the entire methylene blue within a short period of time 95 minuteswhich was a faster rate in comparison to pristine $\mathrm{TiO}^{2}$ nano fibre. An assessment on photo catalytic activity which decreases with the consecutive cycles was brought into reference[48]. Another mechanism that reflects on trapping of electro-hole pairs at the surface of $\mathrm{OH}$ radicals brings the degradation of cationic dyes in solution. The $\mathrm{SnO}_{2}$ QDs fabricated with Mesoporous Silica Nanoparticles (MSN)in relevance to cationic dye behave as a potent adsorptive in the remotion of dye ionsfrom the factory waste water. At the same time, the concentration of ammonia and size of the molecular particle is taken for study specifically with $0.3 \mathrm{~mL}$ of $\mathrm{NH}_{3}$. In the recyclable experiment on dye degradation process $\mathrm{SnO}_{2}$ QDs are found to be very favourable. This process follow pseudo second order kinetics in its techniques and greater negative zeta potential of $-48.05 \mathrm{mV}$ is found in existence of QDs in the bulk of $\mathrm{SiO}_{2}$ material [49].

An another interesting concept in this waste water treatment would be the study of photo catalytic fuel cells which plays an important role in the waste water treatment. In this fuel cells are excellent source for harvesting solar energy is created using $1.2 \mu \mathrm{m} \mathrm{CdS}$ fabricated with $10 \mathrm{~nm}$ $\mathrm{TiO}_{2}$. Light is a source of irradiation of photo anode in this mechanism thereby forming water in the presence of $\mathrm{O}_{2}$ in the waste water and at the same time converting the contaminant into electrical energy or $\mathrm{H}_{2}$ fuel. In such a photo catalytic fuel cell a possibility for formation of electricity was confirmed.The process conformation was enhanced with electrode reaction using acetic acid electrolyte. It is interesting to note that the power density of maximum production accounting to $3980 \mathrm{~mW} / \mathrm{cm}^{3}$ can be produced with enhanced electrolytic conductivity of 63.1 $\mathrm{mS} / \mathrm{cm}[50]$.
An analysis on pharmacompanies on the production of steroid drugs with residual discharge into the water bodies for taken into study. It is because the consumption of such water knowingly or unknowingly by human is life threatening. The solar light intensity of $75.6 \mathrm{k}$ lux is used to degrade these pollutants to $97 \%$. Along with solar cell intensity, $0.25-0.75 \mathrm{~g} / \mathrm{L}$ concentration of catalyst is taken in combination to treat $10-40 \mathrm{mg} / \mathrm{L}$ of pollutant solution which decreases the pollutant effect gradually and consequent cycles extended by adding $0.5 \mathrm{~g} / \mathrm{L}$ of $\mathrm{TiO}_{2}$ QDs. The effective functioning of the catalyst in these depends on the active site with higher concentration of photo catalyst along with $\mathrm{pH}$ range from 3-4.4 in the solution [51].

\section{INORGANIC POLLUTANTS}

The CdSe/CdS core-shell QDs modified with mercapto ethanolas a fluorescence sensor investigate the presence of $\mathrm{Cu}^{2+}$ binds on the surface of the QDs results the displacement reaction with correlation coefficient of 0.9921[52].The defects of organic probe as the fluorescence were overcome by the inorganic probes owing to its rapid response time and stable fluorescence. The capping of CdSMAA (Mercaptoaceticacid) QDs with thiourea detect $\mathrm{Pb}$, $\mathrm{Hg}$ and $\mathrm{Cu}$ ions present in sewer water [53]. Consequently CdTe-MPA ( CdTe-3Mercaptopropionic acid) QDs have excellent photo stability, antiphoto bleaching properties, real time and on-site detection recognize uranyl ions with fluorescent linear concentration range from 0 to $520 \mathrm{nM}$ with $\mathrm{pH}$ 6.5-7.4. MPA was very toxic at low concentration conversely; toxicity was reduced at high concentration [54].Whereas, CdTe/ZnS capped with MPA at $\mathrm{pH} 7.4$ detect copper ions selectively and quantitatively with relative standard deviation $0.23 \%$ and limit of detection 1.5 x $10^{-9}$ M.Thetoxicity of the QDs were limited with aid of $\mathrm{Zn}$ doping[55]. The discharge of uranium and their compounds from nuclear power plants were harmful to living organisms. It was vitally necessary to device an acting technique to recognize and purity the water polluted with the harmful chemicals. Comparatively CdTe-MPA QDs with size $4.2 \mathrm{~nm}$ determine the uranium content in waste water at neutral condition with lower detection limit of $4 \mathrm{~nm}$ than the CdS/MAA QDs. The short time onsite detection shows its ability towards the uranium compounds. Whereas, the negatively charged impurities in lake water decline the recovery rate of uranyl ions[56].

The relatively low electrocatalytic activity of nitrogen doppedGQDs were further enhanced by doping with carbon nano tubes recognized the collection of inorganic pollutants from sewer water in addition with the targeted impurity $\mathrm{Cr}$ (VI) ions. The selective detection of $\mathrm{Cr}$ (VI) ionssolely depend on the $\mathrm{pH}$ of NGQDs[57]. N-doped graphene quantum dots decorated $\mathrm{N}$-doped carbon nanofibers (NGQD@NCNFs) with pyrrolic $-\mathrm{N}$ exhibits electrocatalytic activity with highest quality detection of nitrite ions with detection limit $3 \mu \mathrm{m}$.In a relative manner the particle size was larger than the pristine NGQDs. The dopping of carbon nano fibers with nitrogen speed up the rate of electron transfer.The sensitivity towards the 
hypochlorite was evaluated by comparing the effect of surface coating on different types of QDs. The inert polymer coated QDs possess high sensitivity towards $\mathrm{HClO}$ whereas the reactive coated QDs reduces the sensitivity of $\mathrm{HClO}$.QDs-polyCO${ }_{2}^{-}$was the potential material for the investigation of $\mathrm{HClO}$ than QDs-LACNMe 2 ,QDs-LACNHMeand QDs-poly-NHMe. The exchange of ligands and surfacing does not affect the size of the QDs. Comparatively, alkylamines are better scavenger for $\mathrm{HClO}$ than the thiols [58]. The traces of GO can be recovered using GQDs utilizing ultrasound energy where the $\mathrm{pH}$ was maintained at 7 . Considering the solvent, water shows excellent fluorescence effect than methanol. For the detection of GO the organic impurities from the water should be removed using $\mathrm{NaOH}$ solution[59]. The pristine GQDs detect the $\mathrm{Cu}^{2+}$ ions, in which the metal ions turn off and the biothiol cysteine turn on the Photoluminescence of pristine GQDs [60]. Instead of altering the capping layer the changing of crystal size recognized the ionic impurities from water selectively. GSH (Glutathione), a peptide identifier capped CdTe QDs had no quenching effect on alkali and alkaline earth metal ions [61].

Furthermore, MES-CdSe QDs (2-mercaptoethane sulfonate-CdSe) detect the cyanide anions via partially dynamic quenching, due to its toxicity the $\mathrm{pH}$ was maintained higher than 9.0 [62].The colour of the fluorescence depends on the size of the CdTe QDs, The larger QDs emit red and smaller one emits green fluorescence and the colors can be seen with naked eye. The continuous change of colour was observed with varying concentration of copper ions [63].The quantum yield of N-GQDs were extremely higher than GQDs. The $\pi$-rich chelatorligninsulfonate improve the fluorescence while detecting the $\mathrm{Fe}^{3+}$ ions selectively with limit of detection $0.5 \mathrm{nM}$.The fluorescence effect of SL/GQDs are outstanding in neutral condition, the selective detection was caused by the greater thermodynamic affinity and the quick chelating effect of iron ion with the QDs [64] .The excitation dependent PL was observed in GQDs associated with polyaromatic hydrocarbons and the outstanding behavior was tuning the wavelength and the range of emission wavelength[65].The photoluminescent (PL) GQDs with size 5-10 nm was a potential sensor for $\mathrm{Fe}^{3+}$ and $\mathrm{H}_{2} \mathrm{O}_{2}$. The sensing of $\mathrm{Cr}(\mathrm{VI})$ using $\mathrm{N}-\mathrm{GQD}$ follow the reduction to $\mathrm{Cr}$ (III) [66].The undoped GQDs exhibits surface action for $\mathrm{Fe}^{3+}$ quenching, whereas, the N-GQDs possess static and dynamic quenching[67].

\section{CONCLUSION}

The functioning of Quantum Dots on the investigation of various kinds of contaminant in the wastewater and their performance were discussed. The degradation ability depends on the doping of materials on QDs. Conversely, every technique brings forth excellent result for distinct impurities.

\section{REFERENCES}

[1]. M.Naushad, Surfactant assisted nano-composite cation exchanger: development, characterization and applications for the removal of toxic $\mathrm{Pb}^{2+}$ from aqueous medium. ChemEng J vol. 235, pp.100 108.2014.

[2]. XY.Jiang, GM.Zeng, DL.Huang, Y.Chen, F.Liu, G.H.Huanget al. Remediation of pentachlorophenolcontaminated soil by composting with immobilized Phanerochaetechrysosporium, World MicrobiolBiotechnol, vol. 22(9), pp.909-13.2006.

[3]. V.Matamoros, C.A.Arias, L.X.Nguyen, V.Salvadó\&H.Brix, (2012). Occurrence and behavior of emerging contaminants in surface water and a restored wetland. Chemosphere, vol. 88(9), pp.10831089.2012.

[4]. V.K.Gupta, I.Ali, T.A.Saleh,A.Nayak\&S.Agarwal, Chemical treatment technologies for waste-water recycling - an overview. RSC Advances, 2(16), p.6380, 2012. doi:10.1039/c2ra20340e

[5]. D.Rajkumar, \&K.Palanivelu, (2004). Electrochemical treatment of industrial wastewater. Journal of Hazardous Materials, vol.113(1-3), pp.123-129.2004, doi:10.1016/j.jhazmat.2004.05.039

[6]. J.Geltmeyer, H.Teixido, M. Meire,T.Van Acker, K. Deventer, F.Vanhaecke, K.De Clerck, (2017). $\mathrm{TiO}_{2}$ functionalized nanofibrous membranes for removal of organic (micro)pollutants from water. Separation and Purification Technology, vol.179, pp.533-541, 2017,doi:10.1016/j.seppur.2017.02.037.

[7]. J.Lin, F.Zhang\&Y.Lei, Dietary intake and urinary level of cadmium and breast cancer risk: A meta analysis. Cancer Epidemiology, vol.42, pp.101107. 2016, doi:10.1016/j.canep.2016.04.002.

[8]. M.R.Driss, M.C.Hennion\&M.L.Bouguerraca, Determination of carbaryl and some organophosphorus pesticides in drinking water using on-line liquid chromatographic preconcentration techniques. Journal of Chromatography A, vol. 639(2), pp.352-358, 1993, doi:10.1016/00219673(93)80275-d.

[9]. Remote Sensing Exploration Division ESRIN, “Oil Pollution Monitoring”, ESA Publication Division, http://www.esa. int/esapub/br/br128/br128_1.pdf.

[10]. Y.Cao\&X.Li, Adsorption of graphene for the removal of inorganic pollutants in water purification: a review. Adsorption, vol.20(5-6),pp.713-727. 2014, doi:10.1007/s10450-014-9615-y .

[11]. M.M.Pendergast\&E.M.V.Hoek, A review of water treatment membrane nanotechnologies. Energy \& Environmental Science, vol.4(6), pp.1946. 2011, doi:10.1039/c0ee00541j.

[12]. Coping with Water Scarcity. Challenge of the Twenty-first Century, United Nations (UN) Water. Food and Agricultural Association (FAO), 2007.

[13]. A.P.Alivisatos, W.Gu\&C.Larabell, (2005). Quantum Dots as Cellular Probes. Annual Review of Biomedical Engineering, vol.7(1), pp.55-76, 2005, doi:10.1146/annurev.bioeng.7.060804.100432 . 
[14]. R.C.Ashoori, Electrons in artificial atoms. Nature, vol.379(6564), pp.413-419, 1996, doi:10.1038/379413a0 .

[15]. A.P.Alivisatos, Semiconductor Clusters, Nanocrystals, and Quantum Dots. Science, vol.271(5251), pp.933937, 1996, doi:10.1126/science.271.5251.933.

[16]. A.D.Yoffe, (2001). Semiconductor quantum dots and related systems: Electronic, optical, luminescence and related properties of low dimensional systems. Advances in Physics, vol.50(1), pp.1-208.2001, doi:10.1080/00018730010006608

[17]. A.D.Yoffe, (1993). Low-dimensional systems: quantum size effects and electronic properties of semiconductor microcrystallites (zero-dimensional systems) and some quasi-two-dimensional systems. Advances in Physics, vol.42(2), pp.173-262. 1993, doi:10.1080/00018739300101484.

[18]. S.Gadipelli\&Z.X.Guo, Graphene-based materials: Synthesis and gas sorption, storage and separation. Prog. Mater Sci. vol.69, pp.1-60, 2015.

[19]. A.Tuantranont, Applications of Nanomaterials in Sensors and Diagnostics (Springer-Verlag, 2013).

[20]. J.M.Irudayaraj, Biomedical Nanosensors (Pan Stanford, 2012)

[21]. S.Zhu, Y.Song, X.Zhao, J.Shao, J.Zhang, B.Yang, Nano Res. 8, pp.355,2015.

[22]. S. Zhu, X. Zhao, Y. Song, B. Li, J. Zhang, B. Yang, Graphene Science Handbook, pp.163, 2016.

[23]. S.Zhu, Y.Song, J.Wang, H.Wan, Y.Zhang, Y.Ning\&B.Yang, Photoluminescence mechanism in graphene quantum dots: Quantum confinement effect and surface/edge state. Nano Today, vol.13, pp.10-14. 2017, doi:10.1016/j.nantod.2016.12.006

[24]. F. Wang, Z. Gu, W. Lei, W. Wang, X. Xia and Q. Hao,Graphene quantum dots as a fluorescent sensing platform for highly efficient detection of copper (II) ions,Sens. Actuators, B, vol.190, pp.516-522.,2014.

[25]. J.Ju and W. Chen, Synthesis of highly fluorescent nitrogen-doped graphene quantum dots for sensitive, label-free detection of $\mathrm{Fe}$ (III) in aqueous media, Biosens. Bioelectron, vol. 58, pp.219-225,2014.

[26]. S.Zhu, Y.Song, J.Wang, H.Wan, Y.Zhang, Y. Ning\&B.Yang, (2017). Photoluminescence mechanism in graphene quantum dots: Quantum confinement effect and surface/edge state. Nano Today, vol.13, pp.10-14. 2017, doi:10.1016/j.nantod.2016.12.006.

[27]. K.Tang, V. Baskaran, M.Nemati, Bacteria of the sulphurcycle: an overview of microbiology, biokinetics and their role in petroleum and mining industries. BiochemEng Jvol.44, pp. 73-94), 2009.

[28]. M.N.Hughes,M.N， Centelles,K.P.Moore, (2009) Making and working with hydrogen sulfide. The Chemistry and Generation of Hydrogen Sulfide in Vitro and its Measurement in Vivo: AReview Free Radical Biol Med 47:1346-1353, 2009.

[29]. NutthayaButwang,

SupalaxSrijaranai\&H.T.JohnLuong, Fluorometric determination of hydrogen sulfide via silver-doped $\mathrm{CdS}$ quantum dots in solution and in a test strip.MicrochimicaActa vol.183, pp.1243-1249, 2016.
[30]. R. C. Ashoori, Electrons in artificial atoms. Nature vol.379, pp.413-419, 1996.

[31]. M.A.Gondal, M.A. Dastageer,Spectral red shift in the $\mathrm{Ag}^{2+}$ doped $\mathrm{CdS}$ quantum dots Applied Physics $\mathrm{B}$, Vol.106, Issue 2, pp.419-424,2011. DOI: 10.1007/s00340-011-4679-9.

[32]. MMT.Khan,

R.C.Bhardwaj, C.Bhardwaj,Photodecomposition of $\mathrm{H}_{2} \mathrm{~S}$ by silver doped cadmium sulfide and mixed sulfides with $\mathrm{ZnS}$. Int. J. Hydrogen Energy, vol. 13,pp.7-10, 1988.

[33]. A.P.Alivisatos, W.Gu\&C. Larabell, (2005). Quantum Dots as Cellular Probes. Annual Review of Biomedical Engineering, vol.7(1), pp.5576.2005,doi:10.1146/annurev.bioeng.7.060804.10043 2

[34]. HrvojeKusicab, DanutaLeszczynska, NatalijaKoprivanac, IgorPeternel,Journal of Role of quantum dots nanoparticles in the chemical treatment of colored wastewater: Catalysts or additional pollutants,Environmental Sciences Vol.23, Issue 9, September 2011, pp. 1479-1485, 2011, doi.org/10.1016/S1001-0742(10)60609-2.

[35]. R.Michael,T.Hoffmann Scot, Martin, Wonyong. Choi \&Detlef W. Bahnemann,Environmental Applications of Semiconductor Photocatalysis. Chem. Rev,vol.95, issue.1, pp.69-96, 1995, doi.org/10.1021/cr00033a004.

[36]. S. Fatemeh Seyedpour, Ahmad Rahimpour, Ahmad ArabiShamsabadi, MasoudSoroush. Improved performance and antifouling properties of thin-film composite polyamide membranes modified with nanosized bactericidal graphene quantum dots for forward osmosis, Chemical Engineering Research and Design, Vol.139, pp.321-334, 2018,https://doi.org/10.1016/j.cherd.2018.09.041

[37]. ZhipingZeng, Dingshan Yu, ZimingHe , Jing Liu , Fang-Xing Xiao, Yan Zhang, Rong Wang, Dibakar Bhattacharyya, Timothy Thatt Yang Tan , Graphene Oxide Quantum Dots Covalently Functionalized PVDF Membrane With Significantly-Enhanced Bactericidal and Antibiofouling Performances. SciRep . 2016 Feb 2;6:20142. doi: 10.1038/srep20142.

[38]. Danqing Liu, ZhaozhuZheng, Chaoqun Wang,,Yongqi Yin, Shaoqin Liu, Bin Yang, and Zhaohua Jiang, CdTe Quantum Dots Encapsulated ZnONanorods for Highly Efficient Photoelectrochemical Degradation of Phenols. J. Phys. Chem. C, vol.117, issue.50, pp.26529-26537, 2013. doi.org/10.1021/jp410692y.

[39]. Yong Zhang, Wang and Angelo Mascarenhas. "Quantum Coaxial Cables" for Solar Energy Harvesting ,NanoLett. Vol. 7, issue.5, pp.12641269,2007, https://doi.org/10.1021/n1070174f.

[40]. Xina Wang, Haojun Zhu, YemingXu, HaoWang, Yin Tao, Suikong Hark, Xudong Xiao, and Quan Li, Aligned $\mathrm{ZnO} / \mathrm{CdTe}$ Core-Shell Nanocable Arrays on Indium Tin Oxide: Synthesis and Photoelectrochemical Properties. ACS Nano, vol. 4, issue.6, pp.3302-3308, 2010, doi.org/10.1021/nn1001547. 
[41]. AbdolraoufSamadi-Maybodi, Mohammad-Rasool, Sadeghi-Maleki.SpectrochimicaActaIn-situ synthesis of high stable $\mathrm{CdS}$ quantum dots and their application for photocatalytic degradation of dyes, Part A: Molecular and Biomolecular Spectroscopy Vol.152, issue. 5 pp.156-164, 2016,https://doi.org/10.1016/j.saa.2015.07.052.

[42]. AbdolraoufSamadi-maybodi, FatemehAbbasi, Reza Akhoondi. Aqueous synthesis and characterization of $\mathrm{CdS}$ quantum dots capped with some amino acids and investigations of their photocatalytic activities, Colloids and Surfaces A: Physicochemical and Engineering Aspects Vol.447, Issue.5, pp.111-119. 2014, doi.org/10.1016/j.colsurfa.2014.01.036.

[43]. YumingGuo, Lili Jiang, Lingling Wang, Xiaoman Shi, Qilong Fang, Lin Yang, Fangfang Dong, Chuangchuang Shan. Facile synthesis of stable cadmium sulfide quantum dots with good photocatalytic activities under stabilization of hydrophobic amino acids,Materials Letters Vol.74, Issue.1,pp. 26-29, 2012,https://doi.org/ 10.1016/j.matlet.2012.01.066.

[44]. Xiaoqiang Chen, TuhinPradhan, Fang Wang, Jong Seung Kim, and Juyoung Yoon.Fluorescent Chemosensors Based on Spiroring-Opening of Xanthenes and Related Derivatives,Chem. Rev, vol. 112, Issue. 3, pp. 1910-1956, 2012, doi.org/10.1021/cr200201z.

[45]. Ting-Ting Xu, Jia-Xiang Yang, Ji-Ming Song, JingShuai Chen, He-Lin Niu, Chang-Jie Mao, ShengYiZhang, Yu-HuaShen.Synthesis of high fluorescence graphene quantum dots and their selective detection for $\mathrm{Fe}^{3+}$ in aqueous solution. Sensors and Actuators B: Chemical Vol.243, pp. 863-872,2017, doi.org/10.1016/j.snb.2016.12.048.

[46]. Hengchao Zhang, Hai Ming, SuoyuanLian, Hui Huang, Haitao Li, Lili Zhang, Yang Liu, Zhenhui Kang and Shuit-Tong Lee. $\mathrm{Fe}_{2} \mathrm{O}_{3}$ /carbon quantum dots complex photocatalysts and their enhanced photocatalytic activity under visible light, Dalton Trans.,vol.40, pp.10822-10825,2011.

[47]. Prem Singh Sauda, Bishweshwar Pant, Mira Park, SuHyeongChae, Soo-Jin Park, Mohamed EI-Newehy, Salem S. Al-Deyab, Hak-Yong Kim.Preparation and photocatalytic activity of fly ash incorporated $\mathrm{TiO} 2$ nanofibers for effective removal of organic pollutants,Ceramics International, Vol. 41, Issue 1, Part B, pp.1771-1777, 2015,https://doi.org/10.1016/j.ceramint.2014.09.123.

[48]. DipaDutta,

DinbandhuThakur, DhirendraBahadur. $\mathrm{SnO}_{2}$ quantum dots decorated silica nanoparticles for fast removal of cationic dye (methylene blue) from waste water,Chemical Engineering Journal Vol.281,Issue.1,pp.482490,2015, doi.org/10.1016/j.cej.2015.06.110.

[49]. Bin Wanga, Hao Zhang, Xiao-Ying Lu, Jin Xuanb, Michael K. H. Leunga.Solar photocatalytic fuel cell using $\mathrm{CdS}-\mathrm{TiO}_{2}$ photoanode and air-breathing cathode for wastewater treatment and simultaneous electricity production,Chemical Engineering Journal, Vol.253,
Issue. 1 ,

pp.174-182,

2014 https://doi.org/10.1016/j.cej.2014.05.041

[50]. AmandeepKaur, Ahmad Umar, Sushil Kumar Kansal, Sunlight-driven photocatalytic degradation of nonsteroidal anti-inflammatory drug based on $\mathrm{TiO}_{2}$ quantum dots, Journal of Colloid and Interface Science, Vol.459, 1, pp.257-263,2015,https://doi.org/ 10.1016/j.jcis.2015.08.010.

[51]. Yan Lai, Ying Yu, Ping Zhong, and Jianzhong Wu, Development of Novel Quantum Dots as Fluorescent Sensors for Application in Highly Sensitive Spectrofluorimetric Determination of $\mathrm{Cu}^{2+}$, Analytical Letters, Vol.39, Issue 6, pp.1201-1209,2006.

[52]. R.K. Dutta , Ambika Kumar ,Highly Sensitive and Selective Method for Detecting Ultratrace Levels of Aqueous Uranyl Ions by Strongly PhotoluminescentResponsive Amine-Modified Cadmium Sulfide Quantum Dots,Anal. Chem. Vol.88, Issue.18,pp.90719078, 2016,doi.org/10.1021/acs.analchem.6b01943.

[53]. Sanjeev Kumar Mahto, Chansik Park, Tae HyunYoon, SeogWooRhee, Assessment of cytocompatibility of surface-modified $\mathrm{CdSe} / \mathrm{ZnSe}$ quantum dots for BALB/3T3 fibroblast cells,Toxicology in Vitro, Vol.24, Issue 4, pp.1070-1077, 2010,https://doi.org/10.1016/j.tiv.2010.03.017.

[54]. Wei Bian, Fang Wang, Hao Zhang, Lin Zhang, Li Wang,ShaominShuang,Fluorescent probe for detection of $\mathrm{Cu}^{2+}$ using core-shell CdTe/ZnS quantum dots, Luminescence, Vol.30, Issue7, pp.1064-1070, 2015,https://doi.org/10.1002/bio.2859.

[55]. Xinfeng Chen, Kui Zhang, Huan Yu, Long Yu, HongweiGe, JiYue, TianxinHou, Abdullah M. Asiri, Hadi M. Marwani\&Suhua Wang, Sensitive and selective fluorescence detection of aqueous uranyl ions using water-soluble CdTe quantum dots,Journal of Radioanalytical and Nuclear Chemistry, vol.316, pp.1011-1019,2018, https://doi.org/10.1007/s10967018-5799-z.

[56]. Qing Chen, Yue Hu, Chuangang Hu, Huhu Cheng, Zhipan Zhang,HuiboShaoa andLiangtiQu,Graphene quantum dots-three-dimensional graphene composites for high-performance supercapacitors,Phys. Chem. Chem. Phys., vol.16, pp.19307-19313, 2014,.https://doi.org/10.1039/C4CP02761B

[57]. LiboLi, DongLiu, KunWang, HanpingMao, TianyanYou,Quantitative detection of nitrite with Ndoped graphene quantum dots decorated N-doped carbon nanofibers composite-based electrochemical sensor, Sensors and Actuators B: Chemical, vol.252, pp.17-23,

2017,https://doi.org/10.1016/j.snb.2017.05.155

[58]. Yan Yan, Suhua Wang , Zhongwei Liu, Hongyu Wangand Dejian Huang,CdSe-ZnS Quantum Dots for Selective and Sensitive Detection and Quantification of Hypochlorite,Anal. Chem.vol.82, Issue.23, pp.97759781,2010,https://doi.org/10.1021/ac101929q. 
[59]. Sandra Benítez-Martínez, ÁngelaInmaculadaLópezLorente and Miguel Valcárcel, Graphene Quantum Dots Sensor for the Determination of Graphene Oxide in Environmental Water Samples,Anal. Chem, vol. 86, Issue. 24, pp. 12279-12284,2014 ,https://doi.org/10.1021/ac5035083.

[60]. Xiaofeng Liu , Wei Gao , Xuemei Zhou and Yuanyuan Ma,Pristine graphene quantum dots for detection of copper ions,Journal of Materials Research, Volume 29, pp. 1401-1407, 2014,10.1557/jmr.2014.145

[61]. Lijuan Zhang, ChunliXu\&Baoxin Li, Simple and sensitive detection method for chromium(VI) in water using glutathione-capped CdTe quantum dots as fluorescent probes, MicrochimicaActa, vol.166, pp.6168, 2009, 10.1007/s00604-009-0164-0.

[62]. Wei Jun Jin ,María T. Fernández-Argüelles , José M. Costa-Fernández, Rosario Pereiro and Alfredo SanzMedel,Photoactivated luminescent CdSe quantum dots as sensitive cyanide probes in aqueous solutions, Chem. Commun., pp. 883-885,2005,DOI: 10.1039/B414858D.

[63]. Jianlei Yao, Kui Zhang, Houjuan Zhu, Fang Ma, Mingtai Sun, Huan Yu, Jian Sun, and Suhua Wang,Efficient Ratiometric Fluorescence Probe Based on Dual-Emission Quantum Dots Hybrid for On-Site Determination of Copper Ions,Anal. Chem. Vol.85, Issue.13, 6461-6468, 2013,https://doi.org/10.1021/ac401011r.

[64]. LinaXu, WeiMao, Jinrui Huang, Shouhai Li, Kun Huang, Mei Li, Jianling Xia, Qiang Chen, Economical, green route to highly fluorescence intensity carbon materials based on ligninsulfonate/graphene quantum dots composites: Application as excellent fluorescent sensing platform for detection of $\mathrm{Fe}^{3+}$ ions, Sensors and Actuators B: Chemical, Vol.230, pp. $54-$ 60,2016,https://doi.org/10.1016/j.snb.2015.12.043.

[65]. Li Zhou, JunlongGeng, Bin Liu,Graphene Quantum Dots from Polycyclic Aromatic Hydrocarbon for Bioimaging and Sensing of $\mathrm{Fe}^{3+}$ and Hydrogen Peroxide, Particle, Vol.30, Issue12, pp. 1086-1092, 2013,https://doi.org/10.1002/ppsc.201300170.

[66]. FeiCai, Xidong Liu, Shuang Liu, Hong Liu and Yuming Huang,A simple one-pot synthesis of highly fluorescent nitrogen-doped graphene quantum dots for the detection of $\mathrm{Cr}(\mathrm{VI})$ in aqueous media, $R S C A d v$, vol.4, 52016-52022, 2014, https://doi.org/10.1039/C4RA09320H.

[67]. JianJu, WeiChen,Synthesis of highly fluorescent nitrogen-doped graphene quantum dots for sensitive, label-free detection of $\mathrm{Fe}$ (III) in aqueous media,Biosensors and Bioelectronics, Vol.58,pp. 19225,2014,https://doi.org/10.1016/j.bios.2014.02.061. 